\title{
SIMPLIFIED DYNAMICS OF MULTILAYERED ORTHOTROPIC VISCOELASTIC PLATES $\dagger$
}

\author{
M. A. Biot
}

Avenue Paul Hymans 117, 1200 Brussels, Belgium

\begin{abstract}
Based on a new approach to plate theory, procedures are developed for the dynamic analysis of multilayered plates. They provide analytical simplifications as well as refinements of the physical description which includes the skin effect. The various layers may be anisotropic and each of them may be constituted by thinly laminated materials with stress couples. The damping due to viscoelasticity is evaluated by a method which brings out the effectiveness of each component material. Detailed end conditions may be imposed at the supports at various points across the thickness. It is shown that a plane strain analysis provides immediately solutions of three-dimensional dynamics for multilayered plates with rectangular, triangular and circular plan forms.
\end{abstract}

\section{INTRODUCTION}

THE fundamentals of a new approach to the mechanics of multilayered plates were outlined previously [1] in the context of static problems. The methods may be applied to dynamical problems and provide simplified procedures for the evaluation of natural modes and vibration absorption of multilayered elastic and viscoelastic plates. The layers may be orthotropic. This includes the case where the anisotropy of the individual layers is due to a thinly laminated structure. In addition microelastic properties of such layers are introduced by the use of stress couples.

As already pointed out [1] an adequate theory should take into account the skin effect of anisotropic solid mechanics. On the other hand the evaluation of damping requires improved accuracy of the stress field analysis. This is due to the fact that for heterogeneous matcrials, with uncven distribution of dissipative properties, resonance damping is quite sensitive to localized values of the stress field. Finally the coupling of adhering layers, to be evaluated correctly, should take into account the cross-sectional distortion which is usually overlooked.

While accuracy is thus improved, simplicity of analysis is nevertheless retained. This is in part due to the fact that it is sufficient to solve the problem for plane strain with a sinusoidal distribution along the span. When this has been done solutions are readily obtained for plates with various end conditions and for three-dimensional problems of plates of various plan forms. This leads to the concept of "intrinsic wavelength" defined by the corresponding plane strain solution.

The damping of viscoelastic plates is evaluated by a procedure of linearization which brings out the influence of each individual layer on the overall vibration absorption properties. This is illustrated by treating the single layer and the threelayered plate.

† This research has been sponsored by the A.F. Office of Scientific Research (SREM), 1400 Wilson Boulevard, Arlington, Virginia 22209, through the European Office of Aerospace Research, OAR, United States Air Force, under contract F 61052-69-C-0030. 
For three-dimensional problems the damping is the same or about the same as in the plane-strain solution with the corresponding intrinsic wavelength. Note that the method includes automatically the stretch-bending coupling of plates with asymmetric layers as illustrated for the case of two layers [1].

A considerable refinement is obtained by using exponential branch solutions. This it becomes possible to satisfy very detailed end conditions at the supports. For example we may impose restraints such that zero displacements are prescribed at several points of the end cross-sections. The same refinement is applicable to the edge condition in the three-dimensional problem of a multilayered plate of circular plan form.

\section{BASIC EQUATIONS AND APPROXIMATIONS}

Plane strain in an elastic plate of thickness $h$ is described by the displacements components $u, v$ with the $x$ axis directed along the span and the $y$ axis normal to the plate. The strain components are

$$
e_{x x}=\frac{\partial u}{\partial x} \quad e_{y y}=\frac{\partial v}{\partial y} \quad e_{x y}=\frac{1}{2}\left(\frac{\partial v}{\partial x}+\frac{\partial u}{\partial y}\right) .
$$

We assume the material to be elastically orthotropic with directions of symmetry along $x$ and $y$. If we neglect the stress $\sigma_{y y}$ normal to the plate we may write the stress-strain relations in the simplified form

$$
\begin{aligned}
& \sigma_{x x}=4 M e_{x x} \\
& \sigma_{x y}=2 L e_{x y} .
\end{aligned}
$$

The coefficient $M$ may be expressed by means of the anisotropic elastic coefficients of the material $[1]$. The dynamical equilibrium equations are

$$
\begin{aligned}
& \frac{\partial \sigma_{x x}}{\partial x}+\frac{\partial \sigma_{x y}}{\partial y}=\rho \frac{\partial^{2} u}{\partial t^{2}} \\
& \frac{\partial \sigma_{x y}}{\partial x}+\frac{\partial \sigma_{y y}}{\partial y}=\rho \frac{\partial^{2} v}{\partial t^{2}}
\end{aligned}
$$

where $\rho$ is the mass density. We note that the assumption $\sigma_{y y}=0$ is introduced only in the stress-strain relations (2.2) and not in the equilibrium equations (2.3).

The plate considered here may be inhomogeneous in such a way that it is either continuously or discontinuously stratified. Hence the coefficients $M(y) L(y)$ and the mass density $\rho(y)$ may be functions of $y$.

Let the field be an harmonic function of time and sinusoidally distributed along $x$. Hence we put

$$
\begin{aligned}
& u=U(y) \sin l x \mathrm{e}^{i \alpha t} \\
& v=V \cos l x \mathrm{e}^{i \alpha t} .
\end{aligned}
$$


An additional approximation is introduced here by assuming $V$ to be a constant equal to the average displacement $v$ across the thickness. The second of equations (2.2) yields

$$
\sigma_{x y}=\tau(y) \sin l x \mathrm{e}^{i \alpha t}
$$

with

$$
\tau(y)=L\left(\frac{\mathrm{d} U}{\mathrm{~d} y}-l V\right) .
$$

By eliminating $\sigma_{x x}$ and $U$ between equations (2.2) and the first of equations (2.3) we obtain

$$
\frac{\mathrm{d}}{\mathrm{d} y}\left(\frac{1}{4 \mathfrak{M}} \frac{\mathrm{d} \tau}{\mathrm{d} y}\right)-\frac{l^{2} \tau}{L}=l^{3} V
$$

with

$$
\mathfrak{M}=M\left(1-\frac{\alpha^{2} \rho}{4 M l^{2}}\right)
$$

Let the boundaries of the plate be located at $y= \pm h / 2$ and assume the shear $\tau_{1}=\tau(h / 2)$, $\tau_{2}=\tau(-h / 2)$ to be given at the top and bottom faces. With these boundary conditions the function $\tau(y)$ is obtained by integration of the differential equations (2.7) where $M, L$, $l, \alpha$ and $V$ play the role of parameters.

We finally integrate the second equilibrium equation (2.3) along $y$. We obtain

$$
q=-\int_{-h / 2}^{+h / 2} \tau(y) \mathrm{d} y-\alpha^{2} \rho_{t} V
$$

where

$$
\rho_{t}=\int_{-h / 2}^{+h / 2} \rho(y) \mathrm{d} y
$$

is the total mass per unit area of plate face, while

$$
\left[\sigma_{y y}\right]_{1}-\left[\sigma_{y y}\right]_{2}=q \cos l x \mathrm{e}^{i \alpha t}
$$

represents the total normal load applied to the same unit area. Since $\tau(y)$ is known in terms of $V$, equation (2.9) determines the deflection when the load $q$ is given. When we know $\sigma_{x y}$, the values of $\sigma_{x x}$ and $u$ are determined by combining the first of equations (2.2) with the first of equations (2.3). We obtain

$$
\begin{gathered}
U=\frac{1}{4 \mathfrak{M} l^{2}} \frac{\mathrm{d} \tau}{\mathrm{d} y} \\
\sigma_{x x}=\frac{M}{\mathfrak{M} l} \frac{\mathrm{d} \tau}{\mathrm{d} y} \cos l x .
\end{gathered}
$$

\section{Static analogy}

An important aspect of these results as will appear in the applications resides in the fact that equation (2.7) for $\tau$ and the value (2.12) of $U$ are the same as for the static case 
$(\alpha=0)$ except that $M$ is replaced by the "dynamic" coefficient $\mathfrak{M}$. We also note that

$$
\frac{\alpha^{2} \rho}{4 M l^{2}}=\left(\frac{v_{p}}{v_{M}}\right)^{2}
$$

where $v_{p}=\alpha / l$ is the phase velocity of bending waves in the plate and $v_{M}=2 \sqrt{ }(M / \rho)$ is the velocity of longitudinal compressional waves. In many problems $v_{p} / v_{M} \ll 1$ and we may use the approximation

$$
\mathfrak{M}=M \text {. }
$$

In this case the only difference with the static case resides in the additional term $\alpha^{2} \rho_{t} V$ of equation (2.9).

\section{MULTILAYERED PLATES AND VISCOELASTICITY}

The foregoing results may be used to analyze the plate constituted by a superposition of adherent homogeneous layers. Consider first a single layer of thickness $h$ and constant elastic coefficients $M$ and $L$. The shear stress at the top and bottom of the layer are denoted by $\tau_{1}$ and $\tau_{2}$, respectively. The differential equation (2.7) is readily integrated in this case. Results are formally identical to those for the static case [1]. We find

where

$$
\tau=C_{1} \cosh \beta l y+C_{1} \sinh \beta l y-l L V
$$

$$
\begin{aligned}
C_{1} & =\left[\frac{1}{2}\left(\tau_{1}+\tau_{2}\right)+l L V\right] \frac{1}{\cosh \beta \gamma} \\
C_{2} & =\frac{1}{2}\left(\tau_{1}-\tau_{2}\right) \frac{1}{\sinh \beta \gamma} \\
\gamma & =\frac{1}{2} l h \\
\beta & =2 \sqrt{ }(\mathfrak{M} / L) .
\end{aligned}
$$

From equation (2.12) we derive the values $U_{1}$ and $U_{2}$ of $U$ at the top and bottom of the layer. They are

$$
\begin{aligned}
& U_{1}=\frac{1}{4 l \sqrt{(\mathfrak{M} L)}}\left(\tau_{1} a+\tau_{2} b\right)+c V \\
& U_{2}=-\frac{1}{4 l \sqrt{(\mathfrak{M} L)}}\left(\tau_{1} b+\tau_{2} a\right)-c V
\end{aligned}
$$

where

$$
\begin{aligned}
& a=\tanh \beta \gamma+\frac{1}{\tanh \beta \gamma} \\
& b=\tanh \beta \gamma-\frac{1}{\tanh \beta \gamma} \\
& c=\frac{1}{\beta} \tanh \beta \gamma .
\end{aligned}
$$


The normal l, $d q \cos l x$ applied to this layer is obtained from equation (2.9) we find

$$
q=-\left(\tau_{1}+\tau_{2}\right) c+l^{2} h L V\left(1-\frac{c}{\gamma}\right)-\alpha^{2} \rho h V
$$

For a plate constituted by $n$ adhering layers the $i$ th layer of thickness $h_{i}$ is characterized by coefficients $L_{i}, M_{i}$ and a mass density $\rho_{i}$. Corresponding parameters are $a_{i}, b_{i}, c_{i}, \mathfrak{M}_{i}$. We denote by $\tau_{i}$ and $\tau_{i+1}$ the shear stresses at the top and bottom of the $i$ th layer respectively and by $U_{i}$ and $U_{i+1}$ the displacements at the corresponding faces. The condition of adherence of layers $i$ and $i+1$ are obtained by equating the displacements at the interface. Applying equations (3.3) we derive

where

$$
B_{i} \tau_{i}+\left(A_{i}+A_{i+1}\right) \tau_{i+1}+B_{i+1} \tau_{i+2}=-\left(c_{i}+c_{i+1}\right) l V
$$

$$
A_{i}=\frac{a_{i}}{4 \sqrt{ }\left(\mathfrak{M}_{i} L_{i}\right)} \quad B_{i}=\frac{b_{i}}{4 \sqrt{ }\left(\mathfrak{M}_{i} L_{i}\right)} .
$$

The recurrence equations (3.6) lead to the evaluation of the $n-1$ shear stresses $\tau_{i}$ at the interfaces provided $\tau_{1}$ and $\tau_{n+1}$ at the outerboundaries are given. The values $\tau_{i}$ are found in terms of the single unknown $V$. The latter is evaluated by considering the total load $q \cos l x$ applied to the multilayered plate. It is the sum of the individual loads $q_{i} \cos l x$ acting on each layer. Hence

$$
q=\sum^{i} q_{i}
$$

where according to (3.5)

$$
q_{i}=-\left(\tau_{i}+\tau_{i+1}\right) c_{i}+l^{2} h_{i} L_{i}\left(1-\frac{c_{i}}{\gamma_{i}}\right) V-\alpha^{3} \rho_{i} h_{i} V
$$

We may write

$$
q=-\sum^{i}\left(\tau_{i}+\tau_{i+1}\right) c_{i}+l^{2} K V-\alpha^{2} \rho_{t} V
$$

where

$$
K=\sum h_{i} L_{i}\left(1-\frac{c_{i}}{\gamma_{i}}\right)
$$

and $\rho_{t}=\sum^{i} \rho_{i} h_{i}$ is the total mass per unit area of the plate. Since $\tau_{i}$ is a known function of $V$ while $q$ is given, equation (3.10) determines $V$.

\section{Viscoelastic materials}

Viscoelastic properties of the layers are taken into account by substituting operators for the elastic coefficients $M$ and $L$ in the elastic theory. The general form of these operators was derived from the principles of linear irreversible thermodynamics [2]. They are

$$
\begin{gathered}
\hat{M}=\int_{0}^{\infty} \frac{p}{p+r} M(r) \mathrm{d} r+M^{\prime \prime}+M^{\prime} p \\
\hat{L}=\int_{0}^{\infty} \frac{p}{p+r} L(r) \mathrm{d} r+L^{\prime \prime}+L^{\prime} p
\end{gathered}
$$


where $p=\mathrm{d} / \mathrm{d} t$. For harmonic oscillations $p=i \alpha$ and the operators become complex quantities. In this case we may write

$$
\begin{gathered}
\hat{M}=M+\Delta M \\
\hat{L}=L+\Delta L
\end{gathered}
$$

where $\Delta M$ and $\Delta L$ denote the imaginary parts of $\hat{M}$ and $\hat{L}$.

In practice two important simplifications may be introduced. Since $M$ and $L$ are generally slowly varying functions of the frequency we replace them by constants equal to their values in the vicinity of the significant frequency of the problem. For these real values and assuming $V$ to be known we solve equations (3.6) and (3.10) as in the elastic problem. We then consider the imaginary increments, $\Delta \tau_{i} \Delta q \Delta L, \Delta M$, to be small and linearize all equations for these quantities with the same value of $V$. Using the solution of equations (3.6) and (3.10) we derive the imaginary part $\Delta q$ of $q$. This yields the complex values $q+\Delta q$ of the force required to produce the deflection $V$.

\section{Laminated layers}

The layers themselves may be composed of thinly laminated materials. Such a material may be constituted by repeated groups each of which has a thickness $h^{\prime}$ and contains $k$ layers. The $j$ th has a thickness $h_{j}$ and elastic coefficients $M_{j}$ and $L_{j}$. The laminated material may then be replaced by an anisotropic elastic continuum of coefficients $M, L$, given by

$$
\begin{aligned}
& M=\sum^{j} M_{j} \alpha_{j} \\
& \frac{1}{L}=\sum^{j} \frac{\alpha_{j}}{L_{j}}
\end{aligned}
$$

where $\alpha_{j}$ denotes the fraction of the total thickness $h^{\prime}$ occupied by the $j$ th layer. In addition we must take into account the couple stress i.e. a moment per unit area equal to

$$
\mathscr{M}=b \frac{\partial^{2} v}{\partial x^{2}}
$$

In the present dynamic case the couple stress coefficient may be evaluated exactly as before [1]. The same value is obtained as in the static case

$$
b=\frac{h^{\prime 2}}{3} \sum^{j} M_{j} \alpha_{j}^{3}\left(1-\frac{L}{L_{j}}\right) .
$$

The equilibrium equations (2.3) are also modified by the couple stress and as a consequence equation (3.10) must be replaced by

$$
q=-\sum^{i}\left(\tau_{i}+\tau_{i \mid 1}\right) c_{i}+l^{2} K V-\alpha^{2} \rho_{t} V+l^{4} V \sum^{i} b_{i} h_{i}
$$

where $b_{i}$ is the couple stress coefficient of the $i$ th layer.

This is immediately extended to viscoelastic laminated media replacing the coefficients by the operators $\hat{M}_{i}, \hat{L}_{i}, \hat{b}_{i}$. 


\section{EXAMPLES OF EVALUATION OF DAMPING}

We shall illustrate the method of evaluation of the damping on some specific cases of simply supported plates. Consider the homogeneous anisotropic plate. The span $s$ equal to half the wavelength is

$$
s=\frac{\pi}{l} .
$$

According to the general procedure embodied in equations (3.6) and (3.10) the load $q$ is obtained by using the expression obtained for the static case and adding a dynamic term $-\alpha^{2} \rho_{t} V$. Hence

$$
q=l^{2} h L V\left(1-\frac{\tanh \beta \gamma}{\beta \gamma}\right)-\alpha^{2} \rho_{t} V
$$

Although it is not essential we shall simplify the analysis by assuming $\mathfrak{M}=M$ hence $\beta=2 \sqrt{ }(M / L)$.

For a viscoelastic material $M$ and $L$ are replaced by $M+\Delta M$ and $L+\Delta L$ where $\Delta M$ and $\Delta L$ are the purely imaginary terms. The imaginary part of the load $q$ is represented by $\Delta q$ and evaluated by linearizing equation (4.2) with respect to $\Delta M$ and $\Delta L$. We find

$$
\Delta q=\left(F_{1} \Delta M+F_{2} \Delta L\right) l^{2} h V
$$

with

$$
\begin{aligned}
& F_{1}=\frac{2}{\beta^{2}}\left(\frac{\tanh \beta \gamma}{\beta \gamma}-\frac{1}{\cosh ^{2} \beta \gamma}\right) \\
& F_{2}=1-\frac{3}{2} \frac{\tanh \beta \gamma}{\beta \gamma}+\frac{1}{2} \frac{1}{\cosh ^{2} \beta \gamma}
\end{aligned}
$$

at resonance $q=0$ and the deflection $V$ is entirely due to the load $\Delta q$. Expression (4.3) provides an immediate evaluation of the relative importance of the longitudinal damping $\Delta M$ or the shear damping $\Delta L$ on vibration attenuation. The values of $F_{1}$ and $F_{2}$ depend on the anisotropy as measured by $M / L$ and on thickness to span ratio $h / s=2 \gamma / \pi$. Some numerical values are given in Table 1.

TABLe 1 . VAlues $F_{1}$ AND $F_{2}$ AS FUnCTIONS OF $M / L$ AND $\gamma$

\begin{tabular}{ccccccc}
\hline & $M / L=4$ & & & \multicolumn{3}{c}{$M / L=16$} \\
\cline { 5 - 7 }$\gamma$ & $F_{1}$ & $F_{2}$ & & $\gamma$ & $F_{1}$ & $F_{2}$ \\
\hline 1 & 0.125 & 0.63 & & 0.5 & 0.0625 & 0.63 \\
0.6 & 0.190 & 0.402 & & 0.3 & 0.095 & 0.402 \\
0.3 & 0.192 & 0.210 & & 0.15 & 0.096 & 0.210 \\
0.2 & 1.131 & 0.039 & & 0.10 & 0.065 & 0.039 \\
0.1 & 0.046 & 0.007 & & 0.05 & 0.023 & 0.007 \\
\hline
\end{tabular}

It can be seen that for short spans the shear damping becomes predominant while the opposite is true for large spans.

This result is applicable to the laminated plate where the values of $L$ and $M$ are those 
of the equivalent continuum given by (3.14). In addition we must introduce the complex stress couple coefficient (3.16). The expression (4.3) of $\Delta q$ will then contain an additional term $l^{4} V h \Delta b$ where $\Delta b$ is the imaginary part of the stress couple coefficient (3.16).

As another example we consider the three-layered symmetric plates composed of a core of thickness $h_{2}$ and coefficients $M_{2} L_{2}$ sandwiched between two identical layers of thickness $h_{1}$ and coefficients $M_{1} L_{1}$. Following the static analogy rule we write for the load $q$

$$
\frac{q}{l V}=\frac{8\left(c_{1}+c_{2}\right)^{2}}{a_{1} / \sqrt{ }\left(M_{1} L_{1}\right)+2 \beta_{2} c_{2} / \sqrt{ }\left(M_{2} L_{2}\right)}+2 l h_{1} L_{1}\left(1-\frac{c_{1}}{\gamma_{1}}\right)+l h_{2} L_{2}\left(1-\frac{c_{2}}{\gamma_{2}}\right)-\frac{\alpha^{2} \rho_{t}}{l} .
$$

The first three terms are the same as for the static case derived previously [1]. The resonant frequency is obtained from equation (4.5) by putting $q=0$ and solving for $\alpha$. For simplicity we have introduced the approximation $\mathfrak{M}=M$. The damping is derived by evaluating the imaginary increment $\Delta q$ of $q$ in equation (4.5) due to imaginary increments $\Delta M_{1} \Delta L_{1}$ $\Delta M_{2} \quad \Delta L_{2}$ using linearized expansions with respect to these increments. The result is obtained in the form

$$
\frac{\Delta q}{l V}=\mathscr{A}_{1} \Delta M_{1}+\mathscr{A}_{2} \Delta M_{2}+\mathscr{B}_{1} \Delta L_{1}+\mathscr{B}_{2} \Delta L_{2}
$$

which brings out separately the influence of the longitudinal and shear damping in each of the two materials. The coefficients $\mathscr{A}_{1}, \mathscr{A}_{2}, \mathscr{B}_{1}, \mathscr{B}_{2}$ are easily evaluated functions of $L_{1}$, $M_{1}, L_{2}, M_{2}, h_{1}, h_{2}$ and $l$.

Again the result is applicable to the case where the layers themselves are constituted by two different types of thinly laminated media, using the coefficients of the equivalent anisotropic continuum for each layer and adding on the right side of equation (4.6) the term $\left(2 \Delta b_{1} h+\Delta b_{2} h_{2}\right) l^{3}$ representing the damping due to the stress couples.

The result (4.6) is greatly simplified if the layers are composed of isotropic incompressible materials, in which case we may put

$$
\begin{aligned}
M_{1} & =L_{1}=\mu_{1} \\
M_{2} & =L_{2}=\mu_{2} \\
\beta_{1} & =\beta_{2}=2 .
\end{aligned}
$$

In this case expression (4.5) becomes

$$
\frac{q}{l V}=\frac{\left(\tanh 2 \gamma_{1}+\tanh 2 \gamma_{2}\right)^{2}}{\frac{1}{\mu_{1} \tanh 4 \gamma_{1}}+\frac{\tanh 2 \gamma_{2}}{\mu_{2}}}+2 \mu_{1}\left(2 \gamma_{1}-\tanh 2 \gamma_{1}\right)+\mu_{2}\left(2 \gamma_{2}-\tanh 2 \gamma_{2}\right)-\alpha^{2} \rho_{t} / l
$$

The imaginary part $\Delta q$ of the load is given by

$$
\frac{\Delta q}{l V}=\mathscr{A}_{1} \Delta \mu_{1}+\mathscr{A}_{2} \Delta \mu_{2}
$$




\section{EXAMPLES OF EVALUATION OF DAMPING}

We shall illustrate the method of evaluation of the damping on some specific cases of simply supported plates. Consider the homogeneous anisotropic plate. The span $s$ equal to half the wavelength is

$$
s=\frac{\pi}{l}
$$

According to the general procedure embodied in equations (3.6) and (3.10) the load $q$ is obtained by using the expression obtained for the static case and adding a dynamic term $-\alpha^{2} \rho_{t} V$. Hence

$$
q=l^{2} h L V\left(1-\frac{\tanh \beta \gamma}{\beta \gamma}\right)-\alpha^{2} \rho_{t} V
$$

Although it is not essential we shall simplify the analysis by assuming $\mathfrak{M}=M$ hence $\beta=2 \sqrt{ }(M / L)$.

For a viscoelastic material $M$ and $L$ are replaced by $M+\Delta M$ and $L+\Delta L$ where $\Delta M$ and $\Delta L$ are the purely imaginary terms. The imaginary part of the load $q$ is represented by $\Delta q$ and evaluated by linearizing equation (4.2) with respect to $\Delta M$ and $\Delta L$. We find

$$
\Delta q=\left(F_{1} \Delta M+F_{2} \Delta L\right) l^{2} h V
$$

with

$$
\begin{aligned}
& F_{1}=\frac{2}{\beta^{2}}\left(\frac{\tanh \beta \gamma}{\beta \gamma}-\frac{1}{\cosh ^{2} \beta \gamma}\right) \\
& F_{2}=1-\frac{3}{2} \frac{\tanh \beta \gamma}{\beta \gamma}+\frac{1}{2} \frac{1}{\cosh ^{2} \beta \gamma}
\end{aligned}
$$

at resonance $q=0$ and the deflection $V$ is entirely due to the load $\Delta q$. Expression (4.3) provides an immediate evaluation of the relative importance of the longitudinal damping

\begin{tabular}{|c|c|c|c|c|c|}
\hline \multicolumn{3}{|c|}{$M / L=4$} & \multicolumn{3}{|c|}{$M / L=16$} \\
\hline$\gamma$ & $F_{1}$ & $F_{2}$ & $\gamma$ & $F_{1}$ & $F_{2}$ \\
\hline 1 & 0.125 & 0.63 & 0.5 & 0.0625 & 0.63 \\
\hline 0.6 & 0.190 & 0.402 & 0.3 & 0.095 & 0.402 \\
\hline 0.3 & 0.192 & 0.210 & 0.15 & 0.096 & 0.210 \\
\hline 0.2 & 1.131 & 0.039 & 0.10 & 0.065 & 0.039 \\
\hline $0 \cdot 1$ & 0.046 & 0.007 & 0.05 & 0.023 & 0.007 \\
\hline
\end{tabular}
$\Delta M$ or the shear damping $\Delta L$ on vibration attenuation. The values of $F_{1}$ and $F_{2}$ depend on the anisotropy as measured by $M / L$ and on thickness to span ratio $h / s=2 \gamma / \pi$. Some numerical values are given in Table 1.

Table 1 . Values $F_{1}$ and $F_{2}$ as functions of $M / L$ AND $\gamma$

It can be seen that for short spans the shear damping becomes predominant while the opposite is true for large spans.

This result is applicable to the laminated plate where the values of $L$ and $M$ are those 
of the equivalent continuum given by (3.14). In addition we must introduce the complex stress couple coefficient (3.16). The expression (4.3) of $\Delta q$ will then contain an additional term $l^{4} V h \Delta b$ where $\Delta b$ is the imaginary part of the stress couple coefficient (3.16).

As another example we consider the three-layered symmetric plates composed of a core of thickness $h_{2}$ and coefficients $M_{2} L_{2}$ sandwiched between two identical layers of thickness $h_{1}$ and coefficients $M_{1} L_{1}$. Following the static analogy rule we write for the load $q$

$$
\frac{q}{l V}=\frac{8\left(c_{1}+c_{2}\right)^{2}}{a_{1} / \sqrt{ }\left(M_{1} L_{1}\right)+2 \beta_{2} c_{2} / \sqrt{ }\left(M_{2} L_{2}\right)}+2 l h_{1} L_{1}\left(1-\frac{c_{1}}{\gamma_{1}}\right)+l h_{2} L_{2}\left(1-\frac{c_{2}}{\gamma_{2}}\right)-\frac{\alpha^{2} \rho_{t}}{l} .
$$

The first three terms are the same as for the static case derived previously [1]. The resonant frequency is obtained from equation (4.5) by putting $q=0$ and solving for $\alpha$. For simplicity we have introduced the approximation $\mathfrak{M}=M$. The damping is derived by evaluating the imaginary increment $\Delta q$ of $q$ in equation (4.5) due to imaginary increments $\Delta M_{1} \Delta L_{1}$ $\Delta M_{2} \Delta L_{2}$ using linearized expansions with respect to these increments. The result is obtained in the form

$$
\frac{\Delta q}{l V}=\mathscr{A}_{1} \Delta M_{1}+\mathscr{A}_{2} \Delta M_{2}+\mathscr{B}_{1} \Delta L_{1}+\mathscr{B}_{2} \Delta L_{2}
$$

which brings out separately the influence of the longitudinal and shear damping in each of the two materials. The coefficients $\mathscr{A}_{1}, \mathscr{A}_{2}, \mathscr{B}_{1}, \mathscr{B}_{2}$ are easily evaluated functions of $L_{1}$, $M_{1}, L_{2}, M_{2}, h_{1}, h_{2}$ and $l$.

Again the result is applicable to the case where the layers themselves are constituted by two different types of thinly laminated media, using the coefficients of the equivalent anisotropic continuum for each layer and adding on the right side of equation (4.6) the term $\left(2 \Delta b_{1} h+\Delta b_{2} h_{2}\right) l^{3}$ representing the damping due to the stress couples.

The result (4.6) is greatly simplified if the layers are composed of isotropic incompressible materials, in which case we may put

$$
\begin{aligned}
M_{1} & =L_{1}=\mu_{1} \\
M_{2} & =L_{2}=\mu_{2} \\
\beta_{1} & =\beta_{2}=2 .
\end{aligned}
$$

In this case expression (4.5) becomes

$$
\frac{q}{l V}=\frac{\frac{\left(\tanh 2 \gamma_{1}+\tanh 2 \gamma_{2}\right)^{2}}{\mu_{1} \tanh 4 \gamma_{1}}+\frac{\tanh 2 \gamma_{2}}{\mu_{2}}}{\mu_{2}}+2 \mu_{1}\left(2 \gamma_{1}-\tanh 2 \gamma_{1}\right)+\mu_{2}\left(2 \gamma_{2}-\tanh 2 \gamma_{2}\right)-\alpha^{2} \rho_{t} / l .
$$

The imaginary part $\Delta q$ of the load is given by

$$
\frac{\Delta q}{l V}=\mathscr{A}_{1} \Delta \mu_{1}+\mathscr{A}_{2} \Delta \mu_{2}
$$


with

$$
\begin{aligned}
& \mathscr{A}_{1}=\frac{\mathscr{C}^{2}}{\mu_{1}^{2} \tanh 4 \gamma_{1}}+2\left(2 \gamma_{1}-\tanh 2 \gamma_{1}\right) \\
& \mathscr{A}_{2}=\frac{\mathscr{C}^{2}}{\mu_{2}^{2}} \tanh 2 \gamma_{2}+\left(2 \gamma_{2}-\tanh 2 \gamma_{2}\right) \\
& \mathscr{C}=\frac{\tanh 2 \gamma_{1}+\tanh 2 \gamma_{2}}{\frac{1}{\mu_{1} \tanh 4 \gamma_{1}}+\frac{1}{\mu_{2}} \tanh 2 \gamma_{2}}
\end{aligned}
$$

The intrinsic damping of the materials is measured by the imaginary parts $\Delta \mu_{1}$ and $\Delta \mu_{2}$ of the complex moduli $\hat{\mu}_{1}$ and $\hat{\mu}_{2}$. Expression (4.9) provides an immediate evaluation of the effectiveness of each material in the overall vibration absorption. The coefficients. $\mathscr{A}_{1}$ and $\mathscr{A}_{2}$ depend on the rigidities $\mu_{1}$ and $\mu_{2}$ as well as the wavelength and on the thickness of each layer.

\section{Non sinusoidal loading}

The foregoing results assume a simply supported plate with a loading distributed as a half-sine wave. The method is obviously valid for an arbitrary loading provided the latter is expanded in a series along the span. The results are then applied the various Fourier components with a suitable value of $l$ corresponding to each wavelength.

\section{PLATES WITH BUILT-IN AND OTHER END CONDITIONS}

Until now we have considered a plate of span $s$ simply supported at both ends. However the procedures developed for this case are quite general and may be extended to include very sophisticated end conditions. It is best to illustrate the method by treating first a simple example. We shall consider an homogeneous anisotropic plate without stress couples. $\Lambda$ t both ends of the span $s$ the plate is built-in meaning by this that at these points it is rigidly attached so that the displacement of its faces are both zero.

In order to satisfy such boundary conditions we must consider another type of solution which is exponential along the span instead of sinusoidal. Such solutions are immediately derived from the trigonometric solutions (2.4) and (2.5) if we replace $l$ by $i k$. We write

$$
\begin{aligned}
v & =V \cos i k x \mathrm{e}^{i \alpha t}=V \cosh k x \mathrm{e}^{i \alpha t} \\
u & =i U(y) \sin i k x \mathrm{e}^{i \alpha t}=U(y) \sinh k x \mathrm{e}^{i \alpha t} \\
\sigma_{x y} & =-i \tau(y) \sin i k x \mathrm{e}^{i \alpha t}=\tau(y) \sinh k x \mathrm{e}^{i \alpha t} .
\end{aligned}
$$

As can be seen, in order to obtain real solutions, we must also replace $U$ and $\tau$ by $-i U$ and $-i \tau$, respectively.

The corresponding load distribution is

$$
q \cos i k x \mathrm{e}^{i a t}=q \cosh k x \mathrm{e}^{i \alpha t} .
$$


As a consequence the solution for the homogeneous anisotropic plate is derived quite simply replacing $l$ by $i k$ in equation (4.2). The load is

$$
q=k^{2} h L V\left(\frac{\tanh \frac{1}{2} \beta k h}{\frac{1}{2} \beta k h}-1\right)-\alpha^{2} \rho_{t} V .
$$

Putting $q=0$ in the two equations (4.2) and (5.3) yields the resonant frequency $\alpha$ for the sinusoidal and exponential solutions. We now write the condition that the resonant frequencies are the same for both solutions. This yields the equation

$$
l^{2}\left[1-\frac{\tanh \frac{1}{2} \beta l h}{\frac{1}{2} \beta l h}\right]=k^{2}\left[\frac{\tan \frac{1}{2} \beta k h}{\frac{1}{2} \beta k h}-1\right]
$$

which relates $l$ and $k$. Strictly speaking this equation still contains the unknown $\alpha$ through the parameter $\mathfrak{M}$ as shown by equation (2.8). However as a first approximation we may put $\mathfrak{M}=M$. As the reader can easily ascertain this simplification is not essential since putting $q=0$ in equation (4.2) provides an additional equation for $\alpha$. However numerically the procedure becomes much more cumbersome. In practice it is justified to put $\mathfrak{M}=M$ and if a refinement is needed we may correct the value of $\mathfrak{M}$ after the frequency $\alpha$ has been determined and thus obtain a second approximation.

Under these conditions we may consider (5.4) to be a functional relation defining $k$ as a function of $l$. We may write it in the form

$$
X(X-\tanh X)=Y(\tan Y-Y)
$$

with

$$
X=\frac{1}{2} \beta l h \quad Y=\frac{1}{2} \beta k h .
$$

Numerical values of $Y$ as a function of $X$ are shown in Table 2. The function $Y$ has an infinite number of branches. Only the first two $Y_{1}$ and $Y_{2}$ have been tabulated.

TABLE 2. First two BRANCHES OF THE SOLUtion OF EQUATION (5.5)

\begin{tabular}{lll}
\hline \multicolumn{1}{c}{$X$} & $Y_{1}$ & $Y_{2}$ \\
\hline 0 & 0 & 4.49 \\
0.5 & 0.48 & 4.49 \\
0.8 & 0.70 & 4.50 \\
1.0 & 0.85 & 4.50 \\
1.5 & 1.06 & 4.51 \\
2.0 & 1.22 & 4.52 \\
2.5 & 1.33 & 4.53 \\
3.0 & 1.40 & 4.54 \\
3.5 & 1.44 & 4.56 \\
4.0 & 1.47 & 4.58 \\
\hline
\end{tabular}

Consider now the combination of two solutions, one a sinusoidal solution with $l=2 X / \beta h$, the other an exponential solution corresponding to the first branch $Y_{1}$ with $k_{1}=2 Y_{1} / k h$. The deflection $v$ corresponding to the superposition of these two solutions is

$$
v=V \cos l x+V_{1} \cosh k_{1} x .
$$


For simplicity the factor $\mathrm{e}^{\text {iat }}$ has been omitted. The associated spanwise displacement $u$ is provided by equations (2.4), (2.12) and (3.1), putting $\tau_{1}=\tau_{2}=0$ and substituting suitable imaginary variables for the exponential portion of the solution. This yields

$$
u=V \frac{\sinh \beta l y}{\beta \cosh \frac{1}{2} \beta l h} \sin l x-V_{1} \frac{\sin \beta k_{1} y}{\beta \cos \frac{1}{2} \beta k_{1} h} \sinh k_{1} x .
$$

Denote the span by $s$ and let the origin of $x$ be located at the center of the span. The plate is assumed to be built-in so that the displacements of the faces at the ends are equal to zero. This is expressed by the conditions

$$
u=v=0 \quad \text { for } \quad x= \pm s / 2 \quad y= \pm h / 2 .
$$

Introducing these conditions in equations (5.7) and (5.8) yields

$$
\begin{gathered}
V \cos \frac{1}{2} l s+V_{1} \cosh \frac{1}{2} k_{1} s=0 \\
V \tanh \frac{1}{2} \beta l h \sin \frac{1}{2} l s-V_{1} \tan \frac{1}{2} \beta k_{1} h \sinh \frac{1}{2} k_{1} s=0 .
\end{gathered}
$$

Elimination of $V$ and $V_{1}$ leads to the characteristic equation

$$
\tanh \frac{1}{2} \beta l h \tan \frac{1}{2} l s+\tan \frac{1}{2} \beta k_{1} h \tanh \frac{1}{2} k_{1} s=0 .
$$

Since $k$ is a function of $l$ this determines the unknown $l$. There are an infinite number of roots corresponding to the natural modes of vibration of the plate.

It is interesting to consider the limiting case for large span, hence for small values of $l h$. In this case equation (5.4) is reduced to

$$
l=k_{1}
$$

and the characteristic equation (5.11) becomes

$$
\tan \frac{1}{2} l s+\tanh \frac{1}{2} l s=0
$$

which is equivalent to

$$
\cosh l s \cos l s=1 .
$$

This result coincides with the value obtained from the classical theory of thin plates with built-in ends [3]. The smallest root different from zero is in this case

$$
l s=4.730 \text {. }
$$

Consider a case where the span is not large, for example

$$
\begin{aligned}
s & =5.9 h \\
\frac{M}{L} & =6 .
\end{aligned}
$$

The first characteristic root of equation (5.11) yields the values

$$
\begin{aligned}
l s & =3 \cdot 944 \\
k_{1} s & =2 \cdot 40 .
\end{aligned}
$$


For increasing anisotropy $(M / L \rightarrow \infty)$ the shape of the first mode approaches a half sine wave.

The method outlined here is very general. We may for example satisfy a number of end conditions a lot more detailed than the one assumed here. This can be done by introducing a number of higher branches for the values of $k$. Equations (5.7) and (5.8) are then replaced by

$$
\begin{aligned}
& v=V \cos l x+\sum^{i} V_{i} \cosh k_{i} x \\
& u=V C(y) \sin l x+\sum^{i} V_{i} C_{i}(y) \sinh k_{i} x
\end{aligned}
$$

where the functions $C(y)$ and $C_{i}(y)$ are similar to those in equation (5.8) and $k_{i}$ are the various branch solutions of equation (5.4) as functions of $l$.

Using solutions (5.18) it is possible to derive the natural modes for very complicated boundary conditions. For example it is possible to impose the conditions $u=0 v=0$ of no displacement at several points of the cross section at both ends. This leads to a number of homogeneous equations with an equal number of variables $V$ and $V_{i}$ analogous to equations (5.10). The solution of these equations leads to characteristic values for $l$ and determines the ratios $V_{i} / V$. Hence

$$
V_{i}=R_{i} V
$$

Also other types of conditions may be considered which involve the vanishing of certain stresses components at certain points of the end cross section or even mixed conditions for stress and displacement.

In our example we have assumed the same end conditions at $x= \pm s / 2$, with deflections proportional to $\cos l x$ and $\cosh k x$. Unsymmetric end conditions may of course be treated by adding deflections proportional to $\sin l x$ and $\sinh k x$ easily derived from those of Section 2 by shifting the origin of $x$.

The case of a laminated plate is also analyzed by only a slight change in the analysis. Equation (5.4) is simply replaced by

$$
l^{2}\left(1-\frac{\tanh \frac{1}{2} \beta l h}{\frac{1}{2} \beta l h}\right)+\frac{b l^{4}}{L}=k^{2}\left(\frac{\tan \frac{1}{2} \beta k h}{\frac{1}{2} \beta k h}-1\right)+\frac{b k^{4}}{L}
$$

where $b$ is the stress couple coefficient due to the laminations.

The method is applicable just as readily to the completely general case of plates composed of any number of layers some of which may be constituted by laminated materials with stress couples. The expressions (5.18) for $v$ and $u$ are valid in this case. The function $C(y)$ corresponding to the sinusoidal solutions is derived by solving the recurrence equations (3.6) expressing interfacial adherence. The functions $C_{i}(y)$ corresponding to the exponential solutions are obtained in a similar way by solving the same recurrence equations after replacing $l$ by $i k$ and $\tau_{j}$ by $-i \tau_{j}$. Hence again it is possible to satisfy detailed restraining conditions at the ends of the span by requiring for example that $u$ vanishes at a certain number of points of the end cross-sections. Note that for conditions which are different at each end, we simply need to complete expressions (5.18) by adding terms obtained by interchanging $\sin l x$ with $\cos l x$ and $\sinh k_{i} x$ with $\cosh k_{i} x$. 
Finally we must evaluate the damping for built-in end condition. This may be done by a straight forward procedure which again is applicable to the most general case of a plate with any number of anisotropic layers with stress couples. For simplicity we consider the case with identical boundary conditions at both ends. As we have just remarked this does not restrict the generality. Consider one of the undamped natural mode shapes. The deflection $v(x)$ of this mode may be evaluated as outlined above. The result is

$$
v(x)=V f(x)
$$

where $f(x)$ is of the form

$$
f(x)=\cos l x+\sum^{i} R_{i} \cosh k_{i} x .
$$

Let us evaluate the load distribution required to maintain the deflection (5.21) at a given frequency $\alpha$.

This load distribution is obtained by adding the loads due to each of the terms $\cos l x$ and $\cosh k_{i} x$. They are evaluated from expression (3.17) after suitable changes for the imaginary values associated with the exponential terms. Hence the load distribution is of the form

$$
q(x)=\left[\phi(x)+p^{2} \psi(x)\right] V
$$

where $p=i x$. We choose the amplitudes $V$ of the various modes as generalized coordinates $\mathfrak{q}_{i}$. The corresponding displacements and loads are

$$
\begin{aligned}
& v_{i}(x)=\mathfrak{q}_{i} f_{i}(x) \\
& q_{i}(x)=\mathfrak{q}_{i}\left[\phi_{i}(x)+p^{2} \psi_{i}(x)\right] .
\end{aligned}
$$

The following integral over the span $s$ represents an operational invariant

$$
\mathscr{P}=\frac{1}{2} \int_{-s / 2}^{s / 2} \sum^{i j} v_{i} q_{j} \mathrm{~d} x=\frac{1}{2} \sum^{i j}\left(Z_{i j}+p^{2} m_{i j}\right) q_{i} q_{j} .
$$

The Lagrangian equations of this system may then be written in operational form

$$
\frac{\partial \mathscr{P}}{\partial q_{i}}=Q_{i}
$$

where $Q_{i}$ is the generalized force conjugate to $\mathfrak{q}_{i}$. It is determined by the virtual work of the actual load applied to the plate for a particular variation $\partial \mathfrak{q}_{i}$. Since the generalized coordinates are the natural modes the matrices $Z_{i j}$ and $m_{i j}$ are diagonal. They correspond respectively to the potential and kinetic energies. Until now we have considered an elastic plate. However for viscoelastic materials equations (5.26) remain formally the same. This is a consequence of the general principle of viscoelastic correspondence introduced by the author $[4,5]$ in operator-variational form. In this case the matrix elements become operators $\hat{Z}_{i j}$. They are obtained by substituting operators for the elastic and stress couple coefficients of the various layers constituting the plate. Actually as done above it will be convenient to linearize the operator by writing

$$
Z_{i j}=Z_{i j}+\Delta Z_{i j}
$$


where $\Delta Z_{i j}$ is the imaginary increment expressed as a linear function of the imaginary increments of the operators for the various layers. The operational Lagrangian equations (5.26) are now

$$
\sum^{j}\left(Z_{i j}+\Delta Z_{i j}\right) \mathfrak{q}_{j}+p^{2} \sum^{j} m_{i j} \mathfrak{q}_{j}=Q_{i}
$$

This yields the phase and amplitude of the generalized coordinates $\mathfrak{q}_{i}$ for given applied forces. Since equations (5.28) are in operational form they are well suited to the evaluation of transients, by using the standard procedures of the operational calculus. Near resonance the solution of this system is simplified. Consider for example resonance for the fundamental mode $\mathfrak{q}_{1}$. The first equation (5.28) reduces to

$$
\sum^{j} \Delta Z_{i j} \mathfrak{q}_{j}=Q_{1}
$$

Since the resonant amplitude $q_{1}$ is large compared to the others, equation (5.29) is written approximately

$$
\Delta Z_{11} \mathfrak{q}_{1}=Q_{1}
$$

which yields immediately the resonant amplitude in terms of the applied forces.

\section{EXTENSION TO THREE-DIMENSIONAL DYNAMICS}

We consider a triaxial coordinate system and a multilayered plate of completely general type with layers parallel to the $x z$ plane. The $y$ axis remains normal to the plate. The material of the various layers is assumed to be transverse isotropic. The stress-strain relations for a particular layer are

$$
\begin{aligned}
\sigma_{x x} & =4 M e_{x x}+C e_{z z} \\
\sigma_{z z} & =4 M e_{z z}+C e_{x x} \\
\sigma_{x y} & =2 L e_{x y} \\
\sigma_{y z} & =2 L e_{y z} \\
\sigma_{z x} & =2 L_{12} e_{z x}
\end{aligned}
$$

with

$$
\begin{aligned}
& e_{x x}=\frac{\partial u}{\partial x} \quad e_{z z}=\frac{\partial w}{\partial z} \\
& e_{x y}=\frac{1}{2}\left(\frac{\partial v}{\partial x}+\frac{\partial u}{\partial y}\right) \\
& e_{y z}=\frac{1}{2}\left(\frac{\partial w}{\partial y}+\frac{\partial v}{\partial z}\right) \\
& e_{z x}=\frac{1}{2}\left(\frac{\partial u}{\partial z}+\frac{\partial w}{\partial x}\right) .
\end{aligned}
$$

The displacement components are $u, v, w$. 
Isotropy in the $x z$ plane requires the relation

$$
2 M-\frac{1}{2} C=L_{12} .
$$

The elastic coefficients are functions of $y$.

Suppose we have determined a two dimensional sinusoidal solution such as (2.4) and (2.5). Omitting the time factor $\mathrm{e}^{i \alpha t}$, it may be written

$$
\begin{aligned}
& u=U(y) \sin l x \\
& v=V \cos l x .
\end{aligned}
$$

In this expression $U(y)$ is expressed in terms of $\tau(y)$ by equation (2.12) and $\tau(y)$ itself is obtained by integrating the differential equation (2.7) across the plate.

The corresponding normal load distribution is

$$
q(x)=q \cos l x
$$

where $q$ is given by equation (2.9) or (3.17).

Since the material is transverse isotropic the solution (6.4) remains valid if we give it an angle of rotation $\theta$ around the $y$ axis. This solution is now

$$
\begin{gathered}
u=U(y) \cos \theta \sin (\xi x+\zeta z) \\
w=U(y) \sin \theta \sin (\xi x+\zeta z) \\
v=V \cos (\xi x+\zeta z) \\
q(x, z)=q \cos (\xi x+\zeta z)
\end{gathered}
$$

with

$$
\xi=l \cos \theta \quad \zeta=l \sin \theta .
$$

The solution (6.6) may also be written

$$
\begin{gathered}
u=-\frac{1}{l V} U(y) \frac{\partial u}{\partial x} \\
w=-\frac{1}{l V} U(y) \frac{\partial v}{\partial z} \\
q(x, z)=\frac{q}{V} v .
\end{gathered}
$$

Substitution of the displacements in equations (6.1) and (6.2) yields the corresponding stresses. These relations are invariant for any translation in the $x z$ plane or rotation around the $y$ axis. An important consequence is that they remain valid for the large class of threedimensional solutions obtained by superposition of any number of two-dimensional solutions each of which is given an arbitrary rotation and translation.

\section{Rectangular plates}

A simple application of this result is to plates of rectangular plan form simply supported at the edges. We superpose two of the solutions (6.6) with values $\theta$ and $-\theta$ for the angle 
of rotation. The normal deflection is

$$
v=\frac{1}{2} V[\cos (\xi x+\zeta z)+\cos (\xi x-\zeta z)]=V \cos \xi x \cos \zeta z .
$$

The vertical displacement vanishes for

$$
\xi x= \pm \frac{\pi}{2} \quad \zeta z= \pm \frac{\pi}{2}
$$

This corresponds to rectangular plate of sides $s_{1}$ and $s_{2}$ supported at the edge.

We derive

$$
\xi=\frac{\pi}{s_{1}} \quad \zeta=\frac{\pi}{s_{2}}
$$

and from equation (6.7)

$$
l^{2}=\xi^{2}+\zeta^{2}=\pi^{2} \frac{s_{1}^{2}+s_{2}^{2}}{s_{1}^{2} s_{2}^{2}}
$$

This expresses the wave number $l$ of the basic two-dimensional solution. It may be referred to as the "intrinsic" wave number. The intrinsic wavelength is then $2 \pi / l$.

It is interesting to note that for a square plate $\left(s_{1}=s_{2}\right)$ the intrinsic wavelength is equal to the diagonal $s_{1} \sqrt{ } 2$.

The resonant frequency for the two dimensional solution corresponds to $q=0$. It is the same as for the rectangular plate. Away from resonance $q \neq 0$ and the forced oscillation corresponds to a loading

$$
q(x, z)=q \cos \xi x \cos \zeta z .
$$

We have stated that the solution corresponds to a simply supported plate at the edges, hence free to rotate at these boundaries. That this is a good approximation follows from the fact that the solutions are antisymmetric with respect to the edges. Hence only shear stresses are present at the free edges. It is natural to assume that their cancellation is absorbed almost entirely by the reactions at the support and induces only a negligible plate deflection.

Higher modes of the plate are obtained by considering solutions for which $\xi_{s_{1}}$ and $\zeta s_{2}$ are multiples of $\pi / 2$ as well as solution obtained by translations along $x$ and $z$.

Forced oscillations under arbitrary load distributions may be derived by expending $q(x, z)$ in a double Fourier series and applying the foregoing solutions to each component.

Finally the three-dimensional solutions are valid for viscoelastic materials. We simply substitute in expression (6.13) the corresponding complex value of $q$ of the two-dimensional case.

\section{Triangular plates}

Three-dimensional solutions are similarly obtained for a plate whose edges constitute an equilateral triangle of side $s$ which is simply supported at these edges. We write the two-dimensional deflection as

$$
v=V \sin l x
$$


and evaluate the corresponding loading distribution

$$
q(x)=q \sin l x
$$

where $q$ is evaluated as indicated in Sections 2 and 3.

The two-dimensional solution (6.14) may be written

$$
v=V \sin l \mathbf{n}_{\mathbf{1}} \mathbf{r}
$$

where $\mathbf{n}_{1}$ is a unit vector in the plane $x z$ and $\mathbf{r}$ the coordinate vector in the same plane. It represents a solution which has been rotated so that the crests are normal to $\mathbf{n}_{1}$. We choose three vectors $n_{1} n_{2} n_{3}$ in such a way that

$$
\mathbf{n}_{1}+\mathbf{n}_{2}+\mathbf{n}_{3}=0 .
$$

Hence they constitute an equilateral triangle and are oriented at $120^{\circ}$ relative to each other. We then superpose the three solutions corresponding to $\mathbf{n}_{1}, \mathbf{n}_{2}, \mathbf{n}_{\mathbf{3}}$. We obtain

$$
v(x, z)=V \sin \mathbf{n}_{1} \mathbf{r}+V \sin \mathbf{n}_{2} \mathbf{r}+V \sin \mathbf{l n}_{3} \mathbf{r} .
$$

Using trigonometric identities and taking into account relation (6.17) we may write

$$
v(x, z)=-4 V \sin \left(\frac{1}{2} l_{1} \mathbf{r}\right) \sin \left(\frac{1}{2} \ln _{2} \mathbf{r}\right) \sin \left(\frac{1}{2} \ln _{3} \mathbf{r}\right) .
$$

The corresponding load is

$$
q(x, z)=-4 q \sin \left(\frac{1}{2} l \mathbf{n}_{1} \mathbf{r}\right) \sin \left(\frac{1}{2} l \mathbf{n}_{2} \mathbf{r}\right) \sin \left(\frac{1}{2} l_{3} \mathbf{r}\right) .
$$

The deflection $v(x, z)$ is zero on the three lines

$$
\begin{aligned}
& \frac{1}{2} \ln _{1} \mathbf{r}=\pi \\
& \frac{1}{2} \ln _{2} \mathbf{r}=\pi \\
& \frac{1}{2} \ln _{3} \mathbf{r}=\pi
\end{aligned}
$$

which form an equilateral triangle. Let us orient $\mathbf{n}_{1}$ along the $x$ axis. Then $v=0$ for $x=0$ and $\frac{1}{2} l x=\pi$. Hence the length of the edges of the triangle is

$$
s=\frac{4 \pi}{\sqrt{(3) l}}
$$

Then the intrinsic wavelength is equal to $[\sqrt{ }(3) / 2] s$.

By the same argument as for the rectangular plate it can be seen that the three-dimensional solution (6.18) corresponds to a plate simply supported at the three edges.

For a viscoelastic plate $q$ is replaced by the complex value of the two-dimensional problem of the same intrinsic wavelength.

\section{Circular plates}

The three-dimensional dynamics of a plate of circular plan form may also be derived from two-dimensional solutions. Consider first the sinusoidal solution

$$
v=V \cos (l \mathbf{n r})
$$


where $\mathbf{n}$ is the unit vector in the $x z$ plane. We superpose an infinite number of such solutions by integrating expression (6.22) for all directions of $\mathbf{n}$ in the $x z$ plane. We obtain

$$
v=V \int_{0}^{2 \pi} \cos \left[\operatorname{lr} \cos \left(\theta-\theta_{1}\right)\right] \mathrm{d} \theta
$$

where $r$ is the magnitude of $\mathbf{r}$ and $\theta_{1}$ is the angle between $\mathbf{r}$ and the $x$ axis. The integral is independent of $\theta_{1}$ and equal to

$$
v=V \int_{0}^{2 \pi} \cos [l r \cos \theta] \mathrm{d} \theta .
$$

This is the well-known integral representation of the Bessel function $J_{0}$. Hence

$$
v=2 \pi V J_{0}(l r)
$$

In the present case we also need the exponential solutions represented by

$$
v_{j}=V_{j} \cosh k_{j}(\mathbf{n r}) \text {. }
$$

It is obtained by replacing $l$ by $i k_{j}$ where $k_{j}$ are functions of $l$ representing the various branch solutions of equations such as (5.19).

By the same process of integration for all directions $\mathbf{n}$ in the $x z$ plane we obtain

$$
v_{j}=2 \pi V_{j} I_{0}\left(k_{j} r\right)
$$

where $I_{0}$ is the modified Bessel function. For this type of solution equations (6.8) for the displacements and loads are replaced by

$$
\begin{gathered}
u_{i}=\frac{1}{k_{j} V_{j}} U_{j}(y) \frac{\partial v_{j}}{\partial x} \\
w_{j}=\frac{1}{k_{j} V_{j}} U_{j}(y) \frac{\partial v_{j}}{\partial z} \\
q(x, z)=\frac{q_{j}}{V_{j}} v_{j} .
\end{gathered}
$$

The deflection $v(r)$ obtained by superposition of the solutions (6.25) and (6.27) is

$$
v(r)=V J_{0}(l r)+\sum^{j} V_{j} I_{0}\left(k_{j} r\right) .
$$

The factor $2 \pi$ has been omitted since it may be incorporated in the values of $V$ and $V_{j}$. The corresponding radial displacement $u_{r}$ derive from equations (6.8) and (6.28) is of the form

$$
u_{r}=V C(y) \frac{\mathrm{d} J_{0}}{\mathrm{~d} r}(l r)+\sum^{j} V_{j} C_{j}(y) \frac{\mathrm{d} I_{0}}{\mathrm{~d} r}\left(k_{j} r\right) .
$$

We may impose built-in conditions at the edge. This is expressed by putting $v=u_{r}=0$ at the edge $r=R$ and at certain points of the boundary cross sections i.e. for a number of values of $y$. This leads to homogeneous equations for $V$ and $V_{j}$ and a corresponding characteristic equation for $l$. 
For a simply supported edge we evaluate the radial stress $\sigma_{r r}$ from the displacements using equations (6.1). We then put $v=\sigma_{r r}=0$ for $r=R$ and for a certain number of values of $y$. This again determines the characteristic equation for $l$.

Finally the forced oscillation of the circular plate is evaluated for viscoelastic materials using exactly the same procedure as in the two-dimensional case treated in Section 5.

The simplicity of this procedure which opens the way to the analysis of extremely complex structures may be compared with current procedures $[6,7]$ which in spite of very restrictive assumptions are much more involved analytically.

\section{REFERENCES}

[1] M. A. Bют, A New Approach to the Mechanics of Anisotropic Multilayered Plates. Int. J. Solids Struct. 8, 475-490 (1972).

[2] M. A. Biot, Theory of stress-strain relations in anisotropic viscoclasticity and relaxation phenomena.J. appl. Phys. 25, 1385-1391 (1954).

[3] S. Timoshenko, Vibration Problems in Engineering, p. 232. Van Nostrand (1928).

[4] M. A. Biot, Variational and Lagrangian methods in Viscoelasticity, Deformation and Flow of Solids, IUTAM colloquium, Madrid, 1955, pp. 251-263. Springer (1956).

[5] M. A. Biot, Mechanics of Incremental Deformations. John Wiley (1965).

[6] J. S. KAO and R. J. Ross, Fundamental natural frequencies of circular sandwich plates. AIAA Jnl 7, 2353-2355 (1969).

[7] BOEN-Dar Liaw and R. W. Little, Theory of bending of multilayer sandwich plates. AIAA Jnl 5, 301-304 (1967).

(Received 11 March 1971)

Абстракт-На основе нового подхода к теории пластинок даются способы динамического анализа многослойных пластинок. Они дағот как аналитические упрощения, так и уточнение физиче ской записи, которое учитывает эффект поверхностного слоя. Разные слои могут являтся анизотропными и каждый из них может бытв составленный из пластинчатых материалов, с моментными напряжениями. Определяется затухание, вследствие вязкоупругости, методом показывающим эффективности каждого составленного материала. Подробные краевые условия могут бытв получены при опираниях в разных точках, по толщине. Указывается, что анализ плоского деформированного состояния дает непосредственные решения трехмерной задачи динамики, для многослойных, прямоугольных, трехугольных и круглых пластинок. 